\title{
On the sensitivity of tidal network characterization to power law estimation
}

\author{
M. Jiménez ${ }^{1}$, S. Castanedo ${ }^{1}$, Z. Zhou ${ }^{1}$, G. Coco ${ }^{1}$, R. Medina ${ }^{1}$, and I. Rodriguez-Iturbe ${ }^{2}$ \\ ${ }^{1}$ Environmental Hydraulics Institute "IH Cantabria", Universidad de Cantabria, C/ Isabel Torres no. 5, 39011, \\ Santander, Spain \\ ${ }^{2}$ Department of Civil and Environmental Engineering, Princeton University, Princeton, NJ 08544, USA \\ Correspondence to: M. Jiménez (jimeneztm@unican.es)
}

Received: 11 October 2013 - Revised: 18 December 2013 - Accepted: 21 December 2013 - Published: 1 April 2014

\begin{abstract}
Long-term simulations (3000 yr) of an idealized basin using different tidal ranges $(1,2$ and $3 \mathrm{~m})$ and grain sizes $(120,480$ and $960 \mu \mathrm{m})$ have been performed in order to cover a range of hydrodynamic and sedimentary conditions. Two different cell sizes ( 50 and $100 \mathrm{~m}$ ) have been used to study the impact of cell size on tidal network development. The probability distributions of the drainage area and the drainage volume have been computed for every simulation (during an ebb and a flood phase). Power law distributions are observed in drainage area and drainage volume distribution. As an objective estimation of the exponent of a power law is an open issue, different approaches (linear binning, normalized logarithmic binning, cumulative distribution function and maximum likelihood) proposed by White et al. (2008) to estimate the exponent have been used to carry out a sensitivity analysis. Our findings indicate that although all methods results in high and significant correlation coefficients, more work is needed to develop a universal, objective estimation of the exponent.
\end{abstract}

\section{Introduction}

Channel networks are one of the key elements of a tidal system because they connect the different parts of an estuary with the open sea. A wide variety of network configurations may be found in Nature; each configuration is characterized by specific hydrodynamic, sedimentary and biological characteristics. In this study we specifically focus on "tidal networks" where the riverine or the oceanic forcing are negligible.
Tidal networks are natural systems, and many natural systems present power-law distributions for some of their characteristics. Rivers (Rodriguez-Iturbe et al., 1992) are one example of a natural system where power-law distributions are found. Power laws are signatures of self-similarity or scale invariance. Scale invariance describes behaviours in which the same principles apply or the same processes take place over a wide range of scales.

Several authors have investigated the presence of scale invariances in tidal channel networks. For instance, Rinaldo et al. (1999) looked for scale invariances in the probability distribution of drainage area and drainage volume. These two variables were selected because they have a close relationship to the hydrodynamics and morphodynamics of tidal systems. It must be noted here flora and fauna may also play an important role in some estuaries as shows by many authors (e.g. Fagherazzi et al., 2012; Weerman et al., 2011). We, however, will not be explicitly considering vegetation in our analysis and we only focus on the role of abiotic factors in estuarine dynamics. Future work should analyze if and how vegetation can affect eventually present power laws.

The main objective of this paper is to analyze the probability distributions of drainage area and drainage volume in tidal networks; focusing on the possible existence of scale invariance properties. To achieve this goal and in order to explore the characteristics of different patterns, outputs of several long-term numerical simulations have been used. Variability in numerically simulated tidal networks has been achieved by varying hydrodynamic (tidal range) and sedimentary conditions (mean diameter). Other factors can influence the morphological response of tidal basins (e.g. vegetation, wind waves, cohesive sediments) but their effect is not explored 
in this contribution. We also explore the role that grid resolution plays in pattern formation (by repeating the simulations with 50 and $100 \mathrm{~m}$ grid sizes) and how the numerical grid size can potentially affect to the power law scaling. It must be noted here that when looking for scale invariance properties, the accuracy of the exponent estimation process must be controlled. A sensitivity analysis of the power law exponent to the estimation method is also performed, making use of different methods: linear binning estimation, normalized logarithmic binning (Nlog), cumulative distribution function fit (CDF) and maximum likelihood estimation (MLE).

\section{Methods}

\subsection{Model set-up}

Hydrodynamics and morphodynamics have been simulated with the open source model DELFT3D. DELFT3D solves the estuarine hydrodynamics by means of the non-linear shallow water equations. The hydrodynamic field feeds a sediment transport formulation which leads to morphological change (Lesser et al., 2004). Using a simplified basin geometry similar to the one by Marciano et al. (2005) and Van Maanen et al. (2013), three different tidal ranges (1, 2 and $3 \mathrm{~m}$ ) are combined with three different sediment grain sizes (120, 480 and $960 \mu \mathrm{m}$ ) for a total of nine combination. Each of the nine combinations has been solved for two different grid sizes, $100 \mathrm{~m}$ for the coarse grid and $50 \mathrm{~m}$ for the fine grid, to analyze the impact on the evolution of tidal channels. In all simulations a morphological factor of 200 has been used (Roelvink, 2006). Each simulation covered a span of $3000 \mathrm{yr}$.

\subsection{Probability of drainage area and volume}

Drainage area and drainage volume are the variables that control the size of the channels and the characteristics of the flow in any section of a channel. For this reason we focus our efforts to look for scale invariances in these variables.

Tidal systems are bidirectional and asymmetric; flow during ebb and flood phases is different. For this reason we have analysed the drainage area and volume for each one of the phases. Using the hydrodynamics results, the computation of both variables is carried out in two steps. In the first step, drainage directions are detected from the free surface elevation gradients, which determine flow directions. The drainage direction (the direction in which the flow is moving) of each cell determines the connectivity among cells. In the second step, once cell connectivity has been resolved, the adjacency matrix, containing the connectivity information, is constructed. The adjacency matrix $\left(\mathbf{M}_{i, j}\right)$ is a matrix with dimensions of $n \cdot n$, where $n$ is the number of the vertices in the grid. $\mathbf{M}_{i, j}$, takes the value 1 when a connection from cell $i$ to cell $j$ exists, and 0 otherwise. The adjacency matrix $\left(\mathbf{M}_{i, j}\right)$ is used to compute the drainage area at every point of the basin during a given time instant. However, the adja- cency matrix must correspond to a directed tree, i.e. loops must be removed from cell connectivity. Loops are removed zeroing the elements of $\left(\mathbf{M}_{i, j}\right)$ corresponding to the nodes of the loop. Total drainage area in a cell $i,\left(A_{i}\right)$ is computed after loop removal as follows (Rodriguez-Iturbe and Rinaldo, 1997):

$A_{i}(t)=\sum_{j} \mathbf{M}_{i, j}(t) A_{j}(t)+a_{i}$

The drainage area of each cell $A_{i}$ is computed as the sum of its own area $\left(a_{i}\right)$ plus the area drained by the neighboring cells into the cell under consideration. The drainage volume is computed as the product of the drainage area $A_{i}$ and the corresponding variation of water surface elevation, $\Delta \eta_{i}$.

\subsection{Methods for estimating the exponent of a power law}

Based on the work developed by White et al. (2008), four different approaches have been tested to estimate the exponents of power laws: (1) linear binning. Data are binned into intervals (bins) of equal size $w$, and the histogram of the data is computed. A linear regression is then fitted to the log-transformed values of the probability density function in order to determine the slope $\lambda$ that corresponds to the exponent of the power law. (2) Normalized Logarithmic binning (Nlog). Instead of a constant bin width, a constant bin width in logarithmic space is used. A normalization of the data is carried out to estimate the correct value of the exponent. Differences up to $50 \%$ appear when increasing the total number of bins from 100 to 500 . Here, we tested the estimations with different bin widths to ensure exponent estimations are stable. (3) Cumulative Distribution Function (CDF). The CDF is linearized, by means of a log-transformation, to obtain the exponent $\lambda$ as the slope of a linear regression. (4) Maximum likelihood estimation (MLE). The exponent value is selected as the one that maximizes the likelihood function for the observed sample.

\section{Results}

Numerical simulations show (see Fig. 1) that tidal range and grain size are relevant parameters controlling the formation of patterns of tidal channels. Larger tidal ranges imply larger velocities in the inlet, more sediment is mobilized and channel formation is more rapid and channels are larger. Tidal patterns are also affected by sediment grain sizes and the finer the grain size, the larger the capacity to mobilize sediments. This implies that with finer grain sizes, channel development is faster and more channels develop. Overall, tidal range is directly proportional to the number and size of channels, while sediment grain size is inversely proportional.

Grid size also plays an important role in channel pattern formation. Fig. 1 shows the inner zone of the basin where the tidal network developed. The left-hand side column shows results obtained for the grid size of $50 \mathrm{~m}$, while the right-hand 


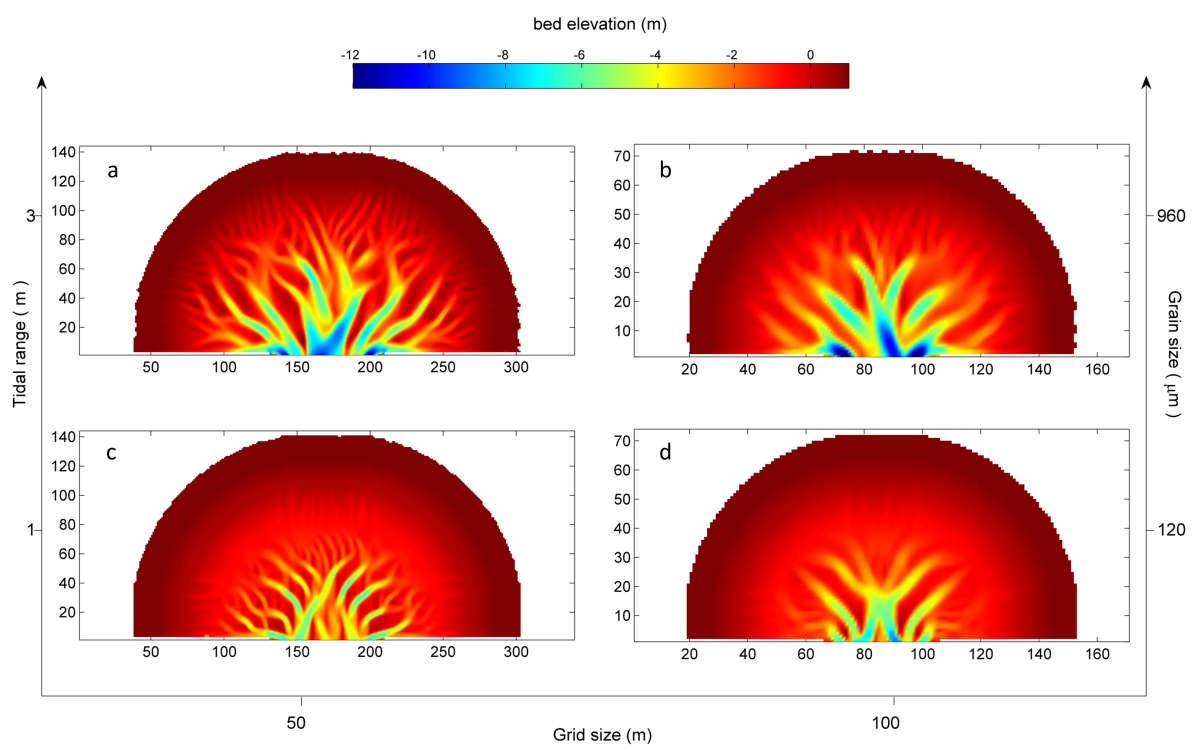

Fig. 1. Numerical simulations in the inner part of the basin using a grid size of $50 \mathrm{~m}$ and $100 \mathrm{~m}$. (a) Simulations with $\mathrm{TR}=3 \mathrm{~m} D_{50}=960 \mu \mathrm{m}$ and grid size $=50 \mathrm{~m}$ (b) with $\mathrm{TR}=3 \mathrm{~m} D_{50}=960 \mu \mathrm{m}$ and grid size $=100 \mathrm{~m}$. (c) Simulations with $\mathrm{TR}=1 \mathrm{~m} D_{50}=120 \mu \mathrm{m}$ and grid size $=50 \mathrm{~m}(\mathbf{d})$ with $\mathrm{TR}=1 \mathrm{~m} D_{50}=120 \mu \mathrm{m}$ and grid size $=100 \mathrm{~m}$.

side column shows the results for the $100 \mathrm{~m}$. The finer the cell size used in the simulations, the greater the number of channels developed. This is simply because when smaller grid sizes are used, smaller scales are resolved. As a consequence, the resulting branching pattern is more detailed and extended.

Drainage area and drainage volume have been computed for every configuration. Figure 2 shows the drainage area and drainage volume for the configuration with tidal range $1 \mathrm{~m}$ and sediment grain size $D_{50}=480 \mu \mathrm{m}$. The red line shows the results for the $50 \mathrm{~m}$ grid size simulations, and the blue one for the $100 \mathrm{~m}$-grid size. Both grid sizes result in similar slopes, but the probabilities associated with drainage area are larger for the fine grid. The finer grid induces the formation of a greater number of narrower channels (see Fig. 1) that occupy a smaller total area. There is then, an increase of the area of the tidal flats that is reflected in the larger area drained in the finer grid. However, Fig. $2 b$ shows that that the total drained volume is larger for the coarser grid. This is due to the fact that the area occupied by the channels is larger in the coarser grid. The average depth is also larger and this induces also a larger total drained volume. Similar behaviour in the distributions has been obtained for the others configurations (not shown).

In order to estimate the exponent of the power laws, we tested four methods (see White et al. 2008). Figure 3 shows an example of the fitting procedure applied to a simulation with tidal range $\mathrm{TR}=3 \mathrm{~m}$ and sediment grain size $D_{50}=960 \mu \mathrm{m}$. Figure $3 \mathrm{a}$ shows the fitting for the drainage area, while Fig. 3b shows the procedure for the drainage volume, in the case of coarse grid size. Data are plotted as grey circles. Correlation analysis shown in Table 1 indicates that the Nlog is marginally superior to the CDF method on average. An analysis of the standard deviation of the estimate shows smaller deviations for the CDF method. Overall, it appears that the CDF method provides more stable estimates, with smaller errors for specific samples, but that the Nlog method provides better fits on average. It is due to the fact that the Nlog approach provides better fitting in the upper part of the distribution where a larger number of data points are concentrated.

Linear binning and MLE methods present lower correlation coefficients; the lowest is for MLE, and slightly larger in the case of linear binning. However, MLE shows standard deviations remarkably lower than the ones computed for CDF and Nlog methods. This is due to the fact that the probability for the smaller areas is very similar in all the sampling distributions obtained because the larger differences appear for the larger areas. As there are many more points in the small areas region, MLE provides more stable estimations and therefore smaller standard deviations. The correlation coefficient is smaller, however, because of the weight differences in the larger areas region have in the computation of the correlation coefficient.

Summarizing, it can be stated that the CDF method provides a good balance between correlation coefficient and stability of the estimate. The Nlog method presents a larger variability, and therefore smaller stability but larger correlations. MLE provides the most stable estimates, adjusting better to the smaller areas region. Linear binning appears as the least accurate of all four methods. 

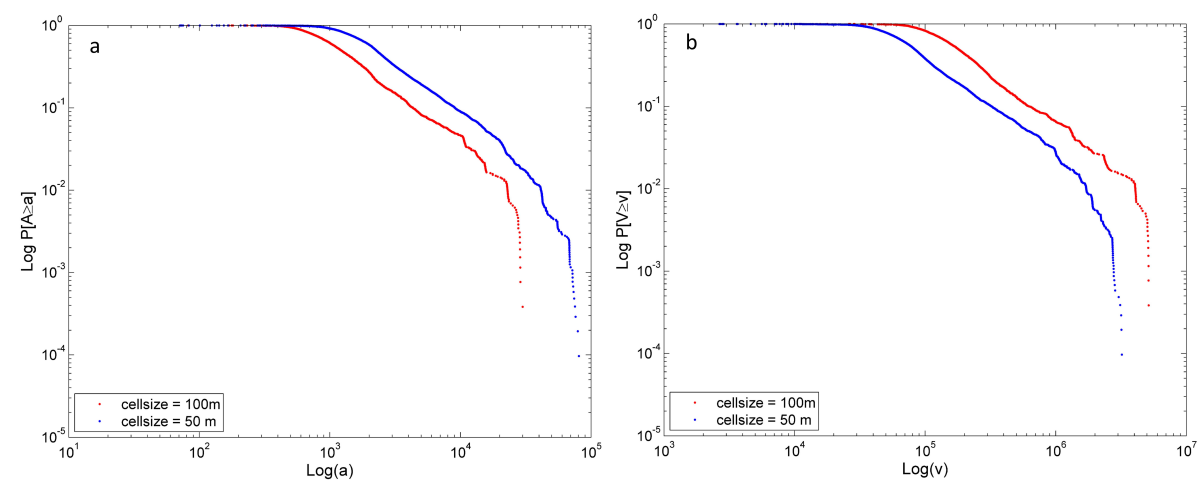

Fig. 2. The prob ability of drainage area and volume, computed for $\mathrm{TR}=1 \mathrm{~m}$ and $D_{50}=480 \mu \mathrm{m}$ during ebbing phase (a) drainage area, (b) drainage volume.

Table 1. Average value of the exponent (considering all tidal ranges and grain sizes), standard deviation obtained and correlation coefficient for the drainage area and drainage volume power laws computed with a grid size of $50 \mathrm{~m}$.

\begin{tabular}{lrrr|rrr}
\hline & \multicolumn{3}{c|}{ Drainage area } & \multicolumn{2}{c}{ Drainage volume } \\
\hline & $\begin{array}{r}\text { Mean exponent } \\
(\lambda)\end{array}$ & $\begin{array}{r}\text { Standard } \\
\text { deviation }(s)\end{array}$ & $\begin{array}{r}\text { Correlation } \\
\text { coefficient }\end{array}$ & $\begin{array}{r}\text { Mean exponent } \\
(\lambda)\end{array}$ & $\begin{array}{r}\text { Standard } \\
\text { deviation }(s)\end{array}$ & $\begin{array}{r}\text { Correlation } \\
\text { coefficient }\end{array}$ \\
\hline Linear binning & $-1,40$ & 0,148 & 0,87 & $-1,04$ & 0,133 & 0,86 \\
Nlog & $-0,96$ & 0,126 & 0,92 & $-0,95$ & 0,12 & 0,91 \\
CDF & $-1,18$ & 0,077 & 0,9 & $-1,16$ & 0,073 & 0,9 \\
MLE & $-1,26$ & 0,016 & 0,89 & $-1,26$ & 0,018 & 0,88 \\
\hline
\end{tabular}

Finally, we have analyzed the influence every parameter (tidal range and sediment grain size) has in the value of the exponent in the power law. Analyzing drainage areas for the case of tidal range of $2 \mathrm{~m}$, a lower value of $1 \mathrm{~m}(50 \%$ reduction) and a higher value of $3 \mathrm{~m}$ ( $50 \%$ increase). The average exponent for the $2 \mathrm{~m}$ case is 1.11 , for the $1 \mathrm{~m}$ case is 1.18 (6.5\% increase) and for the $3 \mathrm{~m}$ is 1.23 (10.59\% increase). Therefore, a decrease of 1 percentage point in tidal range induces an increase of 0.13 percentage points in the value of the exponent. An increase of 1 percentage point in tidal range induces an increase of 0.21 percentage point in the value of the exponent.

In the case of sediment grain size, the middle value is $480 \mu \mathrm{m}$, with a lower value of $120 \mu \mathrm{m},(75 \%$ reduction) and a higher value of $960 \mu \mathrm{m},(100 \%$ increase). The average exponent for $480 \mu \mathrm{m}$, is 1.147 , for $120 \mu \mathrm{m}$, is $1.21(5.72 \%$ increase) and for $960 \mu \mathrm{m}$, is 1.16 (1.24\% increase). A $1 \%$ decrease in sediment grain size induces a 0.01 percentage points increase in exponent value, while a $1 \%$ decrease in sediment grain size induces a 0.08 percentage points increase in exponent value. A similar pattern is found with drainage volumes. We can then conclude that tidal range has a more relevant impact in the final exponent of the power laws that sediment grain size.
Similar conclusions have been extracted in the analysis carried out in the configurations obtained with grid size of $100 \mathrm{~m}$ (not shown).

\section{Conclusions}

Numerical simulations of the development of tidal channel networks showed that tidal channels present self-similar characteristics. Self-similarity is observed in the power law behaviour of the probability distributions of drainage area and drainage volume for ebbing and flooding tides.

Tidal range and sediment grain size are physical parameters with a large impact on the development of tidal networks. However, parameters of the numerical simulation, such as grid size, have also a relevant effect on the final tidal channel network morphology. We have shown that the selection of the grid size is of paramount importance because it does not only affect the final patterns of the channels, it also affects the total drained area and the total drained volume as well as their respective distributions. Further study is required to determine the adequate grid size for different applications. The results presented in this paper are not in line with the conclusions of Rinaldo et al. (1999), where no evidence of self-similarity is found when analyzing four real estuaries. At the same time, we need to point out that our results are not incompatible with Rinaldo et al. (1999) because we found evidence of 

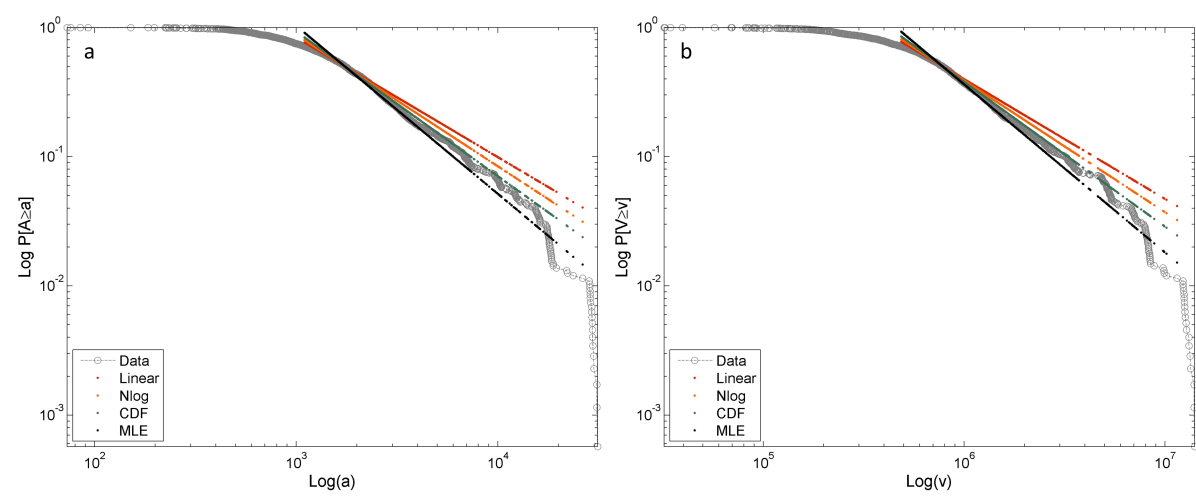

Fig. 3. (a) Probability of drainage area and (b) Probability of volume drainage computed for TR $=3 \mathrm{~m}$ and $D_{50}=960 \mu \mathrm{m}$ with a grid size of $100 \mathrm{~m}$.

self-similarity in the distribution functions of drainage volume and drainage area, a different scaling relationship compared to the one computed by Rinaldo et al. (1999). Moreover, we make use of a detailed flow description to compute the aforementioned functions, which is different from the simplified flow approach of Rinaldo et al. (1999). However, more work is required, for example, to include the effect of vegetation and to test the applicability of our conclusions to real cases.

We have used four different methods to estimate the exponent of the power laws observed in simulated channel networks and compare their performance when characterizing the results of the numerical simulations herein described. We have shown that logarithmic binning and cumulative distribution function fitting provide the most accurate estimates of the four methods. Maximum likelihood provides less accurate which area although very stable ones with respect to changes in the largest regions. All methods results statistically significant in high correlation coefficients although large variability in the exponent of the power law is present. Future studies should attempt to develop a better and possibly universal link between probability distribution and exponent evaluation. Testing using laboratory or field data remains the definitive challenge.

\section{References}

Fagherazzi, S., Kirwan, M. L., Mudd, S. M., Guntenspergen, G. R., Temmerman, S., D’Alpaos, A., Van de Koppel, J., Rybczyk, J. M., Reyes, E., Craft, C., Clough, J.: Numerical models of salt marsh evolution: Ecological and climatic factors, Rev. Geophys., 50, RG1002, doi:10.1029/2011RG000359, 2012.

Lesser, G., Roelvink, J., Vankester, J., and Stelling, G.: Development and validation of a three-dimensional morphological model, Coast. Eng., 51, 883-915, doi:10.1016/j.coastaleng.2004.07.014, 2004.

Marciano, R.: Modeling of channel patterns in short tidal basins, J. Geophys. Res., 110, F01001, doi:10.1029/2003JF000092, 2005.

Rinaldo, A., Fagherazzi, S., Lanzoni, S., Marani, M., and Dietrich, W. E.: Tidal networks 2. Watershed delineation and comparative network morphology, Water Res., 35, 3905-3917, 1999.

Rodríguez-Iturbe, I. and Rinaldo, A.: Fractal River Basins: Chance and Self-Organization, Cambridge, 1997.

Rodríguez-Iturbe, I., Ijjász-Vásquez, E. J., Bras, R. L., and Tarboron, D. G.: Power law distributions of mass and energy in river basins, Water Resour. Res., 28, 1089-1093, doi:10.1029/91WR03033, 1992.

Roelvink, J. A.: Coastal morphodynamic evolution techniques, Coast. Eng., 53, 277-287, doi:10.1016/j.coastaleng.2005.10.015, 2006.

Van Maanen, B., Coco, G., and Bryan, K. R.: Modelling the effects of tidal range and initial bathymetry on the morphological evolution of tidal embayments, Geomorphology, 191, 23-34, doi:10.1016/j.geomorph.2013.02.023, 2013.

Weerman, E. J., Herman, P. M. J., and Van de Koppel, J.: Top-down control regulates self-organization on a patterned intertidal flat, Ecology, 92, 487-495, 2011.

White, E. P., Enquist, B. J., and Green, J. L.: On estimating the exponent of power-law frequency distributions, Ecology, 89, 905912, doi:10.1890/07-1288.1, 2008. 